\title{
A dynamic landscape from femtoseconds to minutes for excess electrons at ice-metal interfaces
}

\author{
U. Bovensiepen ${ }^{1, *}$, C. Gahl ${ }^{2}$, J. Stähler ${ }^{1, \dagger}$, M. Bockstedte ${ }^{3,4}$, M. Meyer ${ }^{1}$, \\ F. Baletto ${ }^{5,}$, S. Scandolo ${ }^{5}$, X.-Y. Zhu ${ }^{6}$, A. Rubio ${ }^{3,7}$, M. Wolf ${ }^{1,8}$ \\ ${ }^{1}$ Fachbereich Physik, Freie Universität Berlin, Arnimallee 14, 14195 Berlin, Germany \\ ${ }^{2}$ Max-Born-Institut für Nichtlineare Optik und Kurzzeitspektroskopie, \\ Max-Born-Str. 2A, 12489 Berlin, Germany \\ ${ }^{3}$ European Theoretical Spectroscopy Facility (ETSF), Departamento Física de Materiales, Edificio \\ Korta, Universidad del País Vasco, Avd. Tolosa 72, 20018 San Sebastián, Spain \\ ${ }^{4}$ Institut für theoretische Festkörperphysik, Universität Erlangen-Nürnberg, \\ Staudtstr. 7/B2, 91058 Erlangen, Germany \\ ${ }^{5}$ The Abdus Salam International Centre of Theoretical Physics (ICTP) and INFM/Democritos \\ National Simulation Center, Strada Costiera 11, 34100 Trieste, Italy \\ ${ }^{6}$ Department of Chemistry, University of Minnesota, Minneapolis, MN 55455, USA \\ ${ }^{7}$ Centro Mixto CSIC-UPV/EHU and Donostia International Physics Center (DIPC) \\ 20018 San Sebastian, Spain \\ ${ }^{8}$ Fritz-Haber-Institut der MPG, Department of Physical Chemistry, Faradayweg 4-6, \\ 14195 Berlin, Germany
}

Stabilization of excess electrons is studied at crystalline ice-metal interfaces by femtosecond time-resolved two-photon photoelectron spectroscopy and ab initio calculations. Following optical excitation into delocalized image potential states, electrons localize at pre-existing defects which are located at the ice-vacuum interface. Remarkably, the stabilization of these trapped electrons is monitored continuously from femtoseconds up to minutes. By first principle calculations we identify defect structures, that support excess electrons in front of crystalline ice surfaces, and suggest that the stabilization proceeds through subsequent conformational substates. The excess electron wave functions are efficiently screened from the underlying bulk and explain the long residence times observed in the experiment. Thereby, we shed light on the collective character of the nuclear rearrangement that determines the energetics over all timescales.

\footnotetext{
*uwe.bovensiepen@physik.fu-berlin.de

${ }^{\dagger}$ present adress: University of Oxford, Department of Physics, Clarendon Laboratory, Parks Road, Oxford OX1 3PU, United Kingdom

ॠFB, Physics Department, King's College London, Strand, WC2R 2LS, London, United Kingdom
} 


\section{Introduction}

Excess electrons in low-dimensional water and ice structures are of general importance in atmospheric science, chemistry, biology, and astrophysics $(1,2,3,4,5)$ since electron-driven excitations and relaxation processes can induce reactions spanning a wide range of energy and time scales (6). In bulk liquid water excess electrons are known to form a solvated complex in which the excess charge is screened (7) and decays by geminate recombination (8). In low dimensional systems like clusters or at ice surfaces the solvation process is determined by the more rigid structure of the water network. In such systems excess electrons can be localized outside the water structure in vacuum $(2,3,9,10)$ where they may interact with a reactant. Generation of excess electrons is achieved in heterogeneous systems like alkali-water anion clusters by charge transfer (11), or photoinduced electron transfer across interfaces $(12,13)$. After photoinjection the excess electron evolves dynamically on an energy landscape due to molecular rearrangement. This landscape is complex, because of the highly polar character and hydrogen bonding of the water network. The corresponding dynamical and structural properties of water are of key relevance for biological processes, as biomolecular systems require water as an active and integral component facilitating functions like protein folding and molecular recognition (14). Dynamical processes in such hydrated macromolecules proceed by transitions among conformational substates (15) which stretch their response times over orders of magnitude $(16,17)$ leading to a pronounced temperature dependence $(18,19)$. Since solvation plays a major role in these processes, it is a central question whether the solvent or the solute are responsible for these dynamics $(14,16,20)$.

Here, we report on the dynamical evolution from femtoseconds ( $\left.1 \mathrm{fs}=10^{-15} \mathrm{~s}\right)$ to minutes of an excess electron in crystalline ice structures adsorbed on a single crystal metal surface. In this system highly localized sites are formed which screen the excess electron efficiently from its environment facilitating its survival over 17 orders of magnitude in time. We show 
that these long-living electrons are localized at the ice-vacuum interface using Xe titration and analyze their stabilization due to molecular rearrangement by time-resolved photoelectron spectroscopy. Performing density functional theory (DFT) calculations, we identify orientational defects or admolecules as initial trapping sites. The calculated defect structures are in agreement with the striking experimental observations of an electron binding site at the ice-vacuum interface that is extremely well screened from the underlying metal substrate and facilitates lifetimes and energetic stabilization up to minutes. We analyze the excess electron-induced structural dynamics on all relevant timescales. These are (i) the excitation and injection of the electron, (ii) its trapping at a preferential site, and (iii) the stabilization by molecular rearrangement until quasi-equilibrium conditions are established. These results have been obtained at crystalline ice structures prepared on a $\mathrm{Ru}(001)$ substrate, which serves both as an electron source and sink in the optically driven interfacial electron transfer.

Interfacial electron transfer (ET) in the strong coupling limit, such as ET between a chemisorbed molecule and a metal surface, occurs within femtoseconds (21) or even attoseconds (22). In this limit, the electron is transferred in a resonant process with a rate governed by the overlap between the acceptor state and the electronic continuum of the metal. If an excess electron is injected from a metal into a molecular adlayer, nuclear rearrangement of molecules due to small polaron formation (23) and electron solvation $(24,25,26)$ leads to screening of the excess charge and to a dynamically decreasing wave function overlap. Consequently, a transition towards a weak electronic coupling limit occurs where electron transfer is controlled by nuclear fluctuations and diffusion processes (27). At metal- or semiconductor-molecule interfaces only a partial screening of the excess electrons has been achieved and the electron residence times have been limited to few picoseconds $(23,24,25,26,27,28)$. An electron injected from a metal into an amorphous ice layer localizes 
and solvates on the fs to ps time scale (24) with a stabilization energy on the order of $100 \mathrm{meV}$ within 500 fs. Similar findings have been reported by Harris (25) and by Petek (26) and their coworkers for adsorbed polar molecules on metal and insulator surfaces, respectively. (See Ref. 29 for a comprehensive review on the topic.) In contrast, we show in the present work that for crystalline $\mathrm{D}_{2} \mathrm{O} / \mathrm{Ru}(001)$ structures the stabilization energy is increased up to several $\mathrm{eV}$ in combination with electron residence times up to minutes.

\section{Experimental Details}

For the preparation of crystalline ice on $\mathrm{Ru}(001)$ we follow established recipes. $\mathrm{D}_{2} \mathrm{O}$ vapor is deposited on $\mathrm{Ru}(001)$ at a substrate temperature of $T=150 \mathrm{~K}$. This is followed by annealing at $162 \mathrm{~K}$ to induce a recrystallization monitored by the isothermal desorption yield. We stop the annealing process when a constant desorption rate is observed and prepare nominal coverages of 2-4 bilayers (BL) (30,31). As illustrated in Fig. 1a, two-photon photoemission (2PPE) uses one photon from an ultrashort laser pulse with a photon energy $h v_{\text {pump }}$ smaller than the work function $E_{\mathrm{vac}}-E_{\mathrm{F}}$ to excite an electron from an occupied state into a bound excited state. A second photon of energy $h v_{\text {probe }}$ photoemits the excited electron, which is analyzed according to its kinetic energy in a time-of-flight spectrometer. Thereby the spectrum of the excited, intermediate states is probed. Using time-resolved 2PPE we probe the transient electron population excited by a UV fs laser pulse of photon energy $h v_{\text {pump }}$ from states below $E_{\mathrm{F}}$ into excited states. A time-delayed probe pulse at $h v_{\text {probe }}$ photoemits these excited electrons and their kinetic energy $E_{\text {kin }}$ is analyzed by a time-of-flight spectrometer (24). The intermediate state energy is given by $E-E_{\mathrm{F}}=E_{\mathrm{kin}}+\Phi-h v_{\text {probe }}$ where $\Phi=E_{\mathrm{vac}}-E_{\mathrm{F}}$ is the work function and $E_{\mathrm{vac}}$ the vacuum level. Pump- and probe pulses with a duration of 50 fs are generated by frequency conversion of the output of a $200 \mathrm{kHz}$ Ti:Sa laser amplifier system by means of optical parametric amplification and subsequent second harmonic generation. Further details are given in Refs. 32,33. 


\section{Long-living excess electrons at the crystalline ice surface}

At metal-molecule interfaces, image potential states (IPS) may exist, which are delocalized parallel to the interface and bound with respect to $E_{\mathrm{vac}}(21)$. Fig. $1 \mathrm{~b}$ shows $2 \mathrm{PPE}$ spectra of crystalline $\mathrm{D}_{2} \mathrm{O} / \mathrm{Ru}(001)$ for different pump-probe delays, which exhibit signatures of the $n=1,2$ IPS with lifetimes of $\tau_{1} \leq 5 \mathrm{fs}$ and $\tau_{2}=50 \mathrm{fs}$, respectively. The spectral feature which we focus on here is observed below $E-E_{\mathrm{F}}=3 \mathrm{eV}$ and cannot be attributed to IPS. After $500 \mathrm{fs}$, its intensity is $\sim 1 \%$ of the IPS at zero delay. Note that the spectra at $0.5 \mathrm{ps}$ (green) and $5 \mathrm{ps}$ (pink) fall on top of each other showing that this feature does not decay on an ultrafast timescale. With increasing time, the decay dynamics slow down dramatically, as the electron energy $\left(E-E_{\mathrm{F}} \approx 2 \mathrm{eV}\right)$ and population are nearly constant from 0.5 ps to $5 \mathrm{ps}$. We label these long-living, trapped electrons by $\mathrm{e}_{\mathrm{T}}$. Fig. 1c shows the complete spectral signature of $\mathrm{e}_{\mathrm{T}}$. Furthermore, it demonstrates that these electrons decay and stabilize on macroscopic timescales of minutes which is the central topic of this paper. In order to follow these slow processes further details on the experimental approach and the $\mathrm{e}_{\mathrm{T}}$ signature are required which we present in the following before we turn to the underlying processes. 

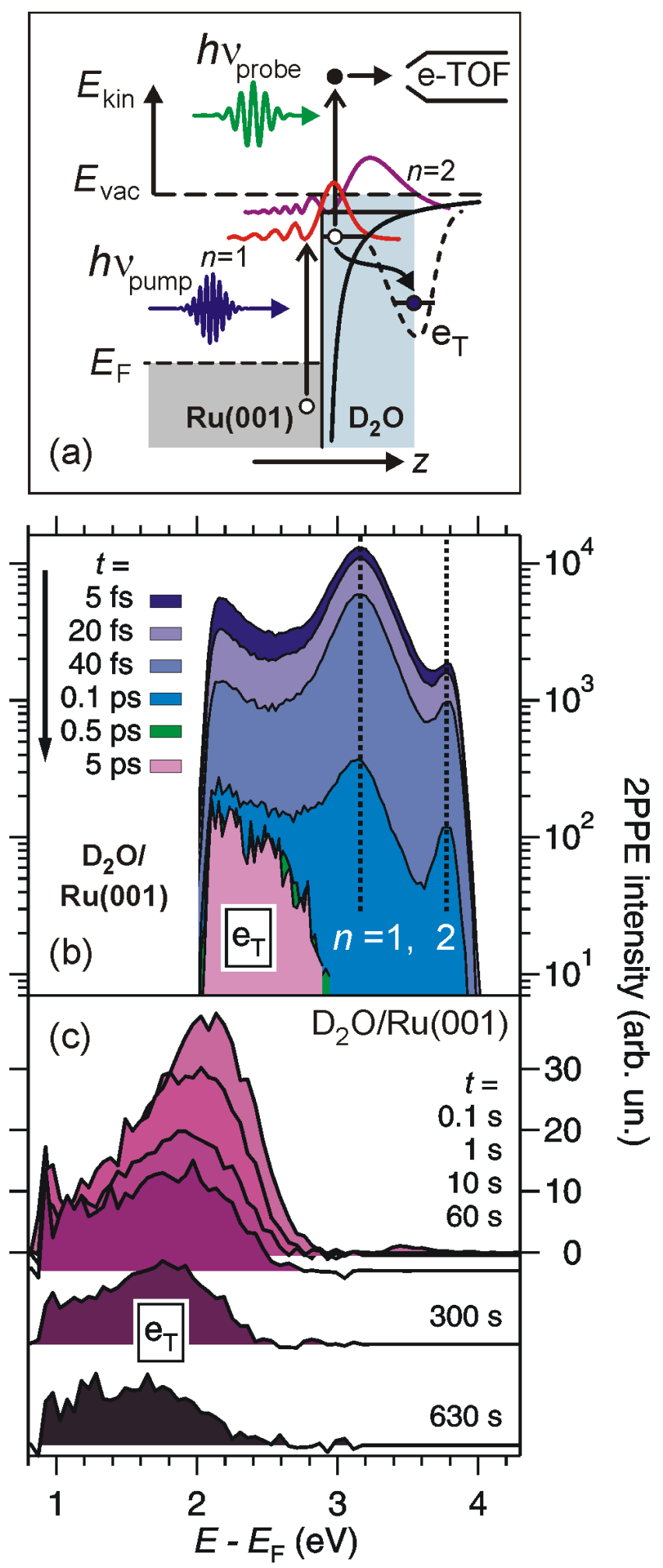

Fig. 1: (a) Time-resolved 2PPE spectroscopy; (b) 2PPE spectra at indicated pump-probe delays employing fslaser pulses with $h v_{\text {pump }}=3.9 \mathrm{eV}, h v_{\text {probe }}=1.95 \mathrm{eV}$ of $60 \mathrm{fs}$ duration on a $2 \mathrm{BL}$ crystalline $\mathrm{D}_{2} \mathrm{O}$ adlayer on $\mathrm{Ru}(001)$ at $T=43 \mathrm{~K}$. The low energy cut-off at $2 \mathrm{eV}$ originates from the probe photon energy that probes electrons down to $E_{\mathrm{vac}}-h v_{\text {probe }}$, but not below. Delay-independent contributions have been subtracted from the spectra. (c) $2 \mathrm{PPE}$ spectra with time delays of seconds (see text), $h v_{\text {pump }}=3.9 \mathrm{eV}, h v_{\text {probe }}=3.1 \mathrm{eV}$. 
Note that only part of the $\mathrm{e}_{\mathrm{T}}$ peak is probed in the two-color pump-probe scheme used in Fig. $1 \mathrm{~b}$ due to the limited energy of the visible probe photon. To reveal the complete $\mathrm{e}_{\mathrm{T}}$ peak and to identify the photoexcitation mechanism, we show in Fig. 2 and Fig. 3 single color 2PPE spectra. Thereby, one UV photon populates $\mathrm{e}_{\mathrm{T}}$, and a second UV photon of equal energy emits the electron for detection. The $2 \mathrm{PPE}$ spectra of crystalline $\mathrm{D}_{2} \mathrm{O} / \mathrm{Ru}(001)$ in Fig. 2 present distinct features: These are the low energy cut-off, a d-band surface resonance of $\mathrm{Ru}(001)$ just below $E-E_{\mathrm{F}}=1 \mathrm{eV}$, a pronounced peak $2 \mathrm{eV}$ above $E_{\mathrm{F}}$, presenting the trapped electrons $\mathrm{e}_{\mathrm{T}}$ and has not been reported for amorphous ice films $(24,34)$, and the first image potential state (IPS) of the $\mathrm{D}_{2} \mathrm{O}-\mathrm{Ru}$ interface at $3.2 \mathrm{eV}$. The d-band resonance (35) and the IPS (36) are intermediate states in the $2 \mathrm{PPE}$ process with sub 5 fs lifetimes. Thus, $2 \mathrm{PPE}$ from these states is a second order process and the respective intensity scales with the power density of the UV laser pulse. 2PPE spectra shown in Fig. 2 have been recorded at $T=43 \mathrm{~K}$ for different UV pulse durations between 100 and 300 fs, i.e. for different power density. Analysis of different features in the 2PPE spectra on the laser pulse duration reveals that population and photoemission for $\mathrm{e}_{\mathrm{T}}$ occur in fact by subsequent laser pulses separated by at least once the inverse laser repetition rate $1 / \rho=5 \mu \mathrm{s}$. As expected, an increase of the $2 \mathrm{PPE}$ yield is observed for decreasing pulse duration (i.e. increasing power) in case of the IPS, the $\mathrm{d}$-band resonance and the low energy cut-off. However, the $\mathrm{e}_{\mathrm{T}}$ contribution is unaffected by the change of pulse duration. This effect can be explained exclusively by lifetimes of $\mathrm{e}_{\mathrm{T}}$ electrons which exceed the inverse laser repetition rate of $5 \mu \mathrm{s}$. The $\mathrm{e}_{\mathrm{T}}$ state is thus populated by a first laser pulse and electrons from $\mathrm{e}_{\mathrm{T}}$ are photoemitted by subsequent pulses so that excitation of and photoemission from that state are not correlated. In other words, the $\mathrm{e}_{\mathrm{T}}$ spectrum represents a photo-stationary state, whereby the photo-induced population of the long-living intermediate state $2 \mathrm{eV}$ above $E_{\mathrm{F}}$ and its depopulation are balanced. 


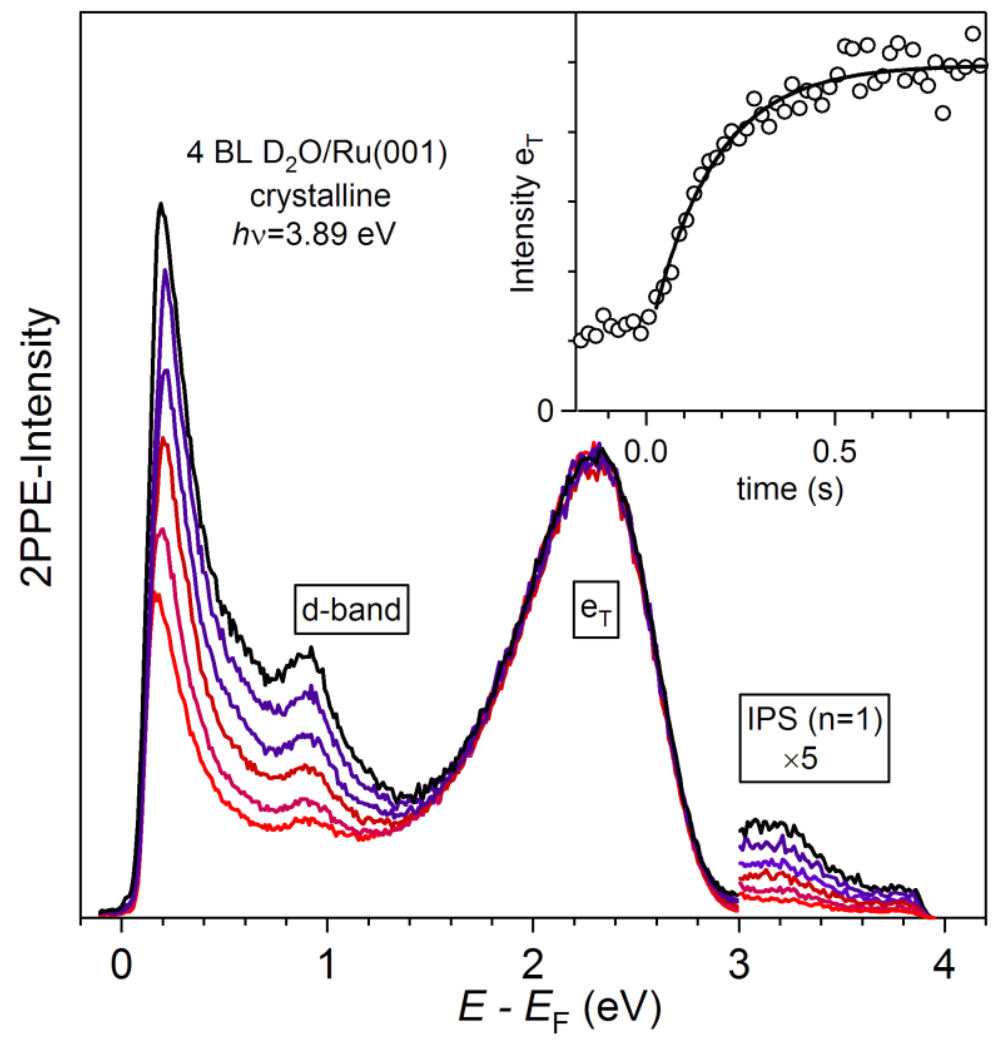

Fig. 2: Single color two-photon photoemission spectra of a crystalline $\mathrm{D}_{2} \mathrm{O}$ layer of 4 bilayers coverage on $\mathrm{Ru}(001)$ are given for different laser pulse durations between $100 \mathrm{fs}$ (black, top) and $300 \mathrm{fs}$ (red, bottom) at $T=30 \mathrm{~K}$. The spectra exhibit three features (low energy cut-off, d-band, and $n=1$ image potential state) that vary with the inverse square of the laser pulse duration and one $\left(\mathrm{e}_{\mathrm{T}}\right)$ that is independent of the pulse duration. The intensity of the latter builds up, as shown in the inset, by formation of a photo-stationary state when population and depopulation have equilibrated with a time constant of $0.17 \mathrm{~s}$ (solid line).

Under the employed absorbed fluence of $0.02 \mathrm{~mJ} / \mathrm{cm}^{2}$ formation of the photo-stationary state measured by the $2 \mathrm{PPE}$ intensity build-up of $\mathrm{e}_{\mathrm{T}}$ in real time - occurs with a time constant of $0.17(1) \mathrm{s}$ as shown in the inset of Fig. 2. From a rate equation analysis we have estimated the density of sites contributing to these long-living states to be on the order of $10^{-4}$ per ice lattice site (32). In this analysis, we made the assumption that the population decay due to electron transfer back to the metal can be neglected in comparison to the photo-induced depopulation of $\mathrm{e}_{\mathrm{T}}$ sites and that irradiation occurs continuously. In view of the extremely long lifetimes observed for $\mathrm{e}_{\mathrm{T}}$ these assumptions are justified. Thus, there must be a minority species like 
defects at the crystalline ice surface that provide trapping sites for these extremely long-living electrons ( $>2 \mathrm{eV}$ above $\left.E_{\mathrm{F}}\right)$ at $\mathrm{nm}$ distance in front of the metal surface.

Comparing to the fs two-color spectrum (Fig. 1b), the $\mathrm{e}_{\mathrm{T}^{-}}$and the $n=1$ peak are by the single color 2PPE experiment completely resolved. Furthermore, the $\mathrm{e}_{\mathrm{T}}$ feature is more intense than the $n=1$ IPS because of the long lifetime. An electron in the $\mathrm{e}_{\mathrm{T}}$ state can be photoemitted by any subsequent laser pulse while an electron in the ultrashort-lived IPS is probed within the same laser pulse.

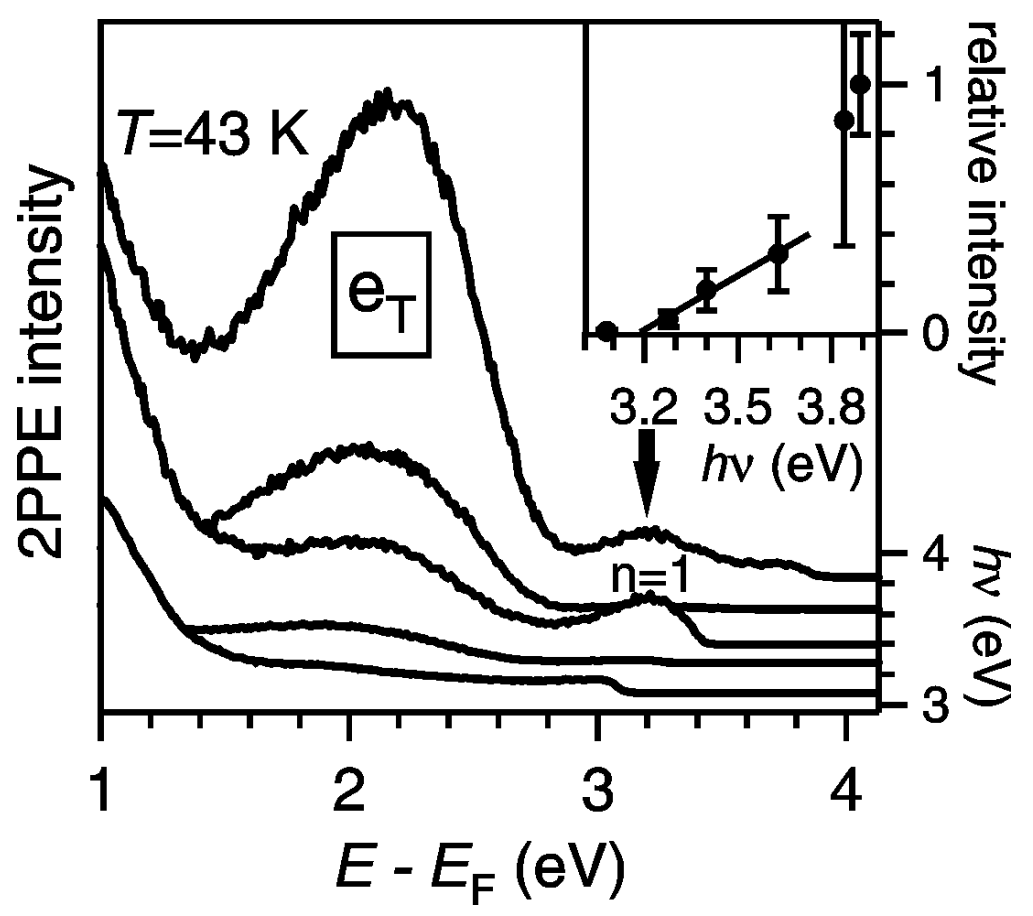

Fig. 3: Single color 2PPE spectra taken at $35 \mathrm{~K}$ for different UV photon energies. The 2PPE intensity has been normalized to the photon density in the laser pulses. The inset shows the intensity of the $\mathrm{e}_{\mathrm{T}}$ peak normalized to the intensity for $h v=3.9 \mathrm{eV}$.

To analyze the photo-injection process we have varied the photon energy $h v_{\text {pump. }}$. Respective 2PPE spectra are given in Fig. 3 and the inset depicts the intensity of the $\mathrm{e}_{\mathrm{T}}$ peak as a function of $h v_{\text {pump. }}$. We find a clear threshold of $h v_{\text {pump }}=3.2 \mathrm{eV}$ necessary to generate electrons in $\mathrm{e}_{\mathrm{T}}$, which coincides with the energy of the $n=1$ IPS (Fig. 1b, Fig. 3). We conclude the following excitation mechanism (Fig. 1a): For $h v_{\text {pump }} \geq 3.2 \mathrm{eV}$ electrons are 
injected into the laterally delocalized $n=1$ IPS from where they can get trapped in the ice. Because the observed lifetime of the $n=1$ IPS is $\leq 5$ fs (36), the electrons are trapped in preexisting defects of the ice structure as a dynamic polaron formation would take longer time (23). The localized nature of these excess electrons has been verified by angle-dependent 2PPE experiments. As shown in Fig. 4 the energy of the $\mathrm{e}_{\mathrm{T}}$ feature does not disperse when the photoemission angle with respect to the surface is varied, which is a direct consequence of the localized electronic wave packet (23). We find a small shift of the peak maximum to lower kinetic energy with increasing angle, which cannot be explained with a delocalized state. The weak negative dispersion has been observed before for localized electronic states at amorphous ice-metal interfaces and characterizes the stabilization of a localized electron due to interaction with the surrounding water dipoles (33).

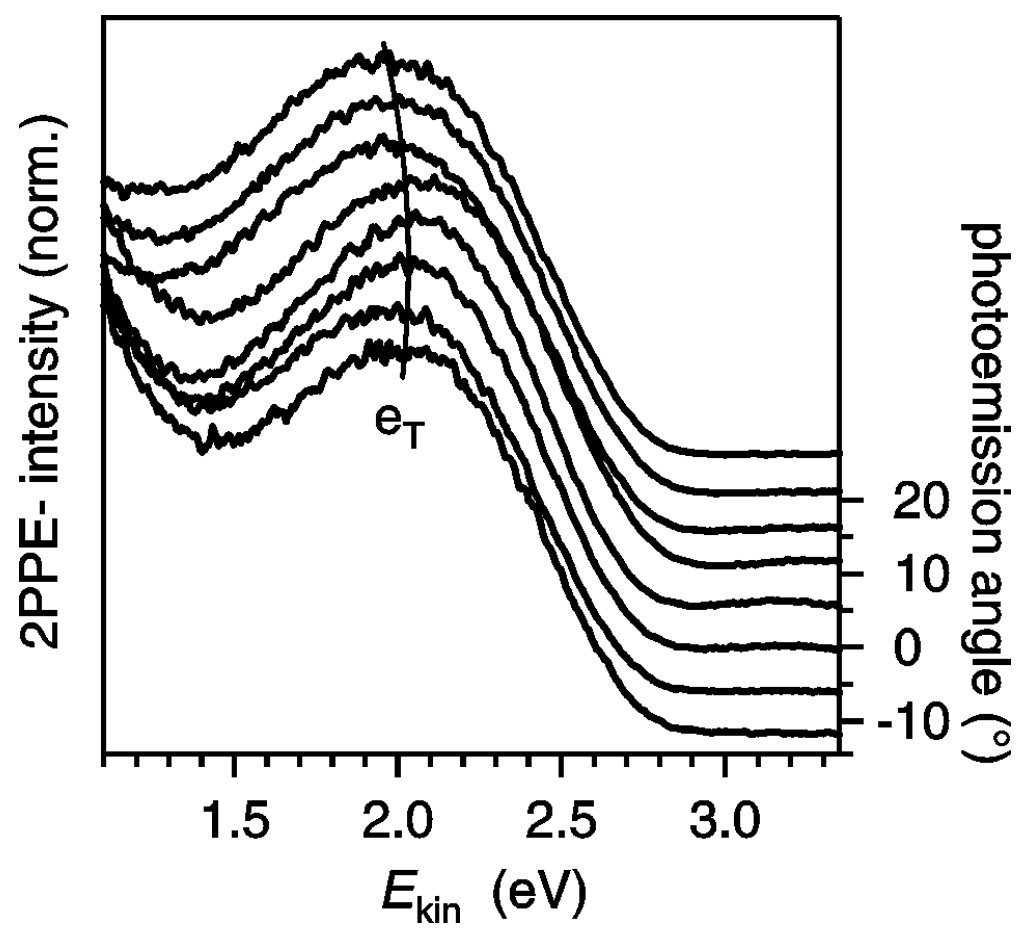

Fig. 4: Angle-dependent 2PPE spectroscopy for the long-living electron feature at $2 \mathrm{eV}$ above $E_{\mathrm{F}}$. The solid line guides the eye to the peak maxima. Peak maxima have been normalized to the one detected in normal emission. Angle-dependent spectra have been measured by rotation of the sample. Vacuum levels of sample and spectrometer have been aligned for every angle individually by applying a bias voltage to the sample. Thereby we compensate for dispersion effects in the vacuum energy as a function of angle and $E_{\text {kin }}$ is well defined for all photoemission angles. 
Due to the long-living nature of $\mathrm{e}_{\mathrm{T}}$ electrons their full relaxation cannot be observed in an ordinary pump-probe experiment. To follow this slow process, we do not excite this state by a single laser pulse, but with subsequent pulses. Since the lifetime of $\mathrm{e}_{\mathrm{T}}$ is larger than the laser repetition time $1 / \rho$, the population of $\mathrm{e}_{\mathrm{T}}$ builds up over several pulses. Simultaneously, $\mathrm{e}_{\mathrm{T}}$ electrons are photoemitted and decay. Thus, population and depopulation compete with each other leading to the photo-stationary state introduced in Fig. 2. Time-resolved spectra in Fig. 1c are recorded by generating the photo-stationary state with $h v_{\text {pump }}$, waiting for the desired delay time with the sample in the dark, and photoemitting the population of $\mathrm{e}_{\mathrm{T}}$ with visible pulses $h v_{\text {probe. }}$ As shown in Fig. 1c this pump-probe scheme allows us to probe dynamics up to $630 \mathrm{~s}$. We observe a continuous increase of the $\mathrm{e}_{\mathrm{T}}$ binding energy together with a decrease in population.

The extremely long lifetime of the $\mathrm{e}_{\mathrm{T}}$ peak appears unexpected because of our previous findings for amorphous ice $(24,27,34)$ and the electron's proximity to the metal surface where $\sim 2 \mathrm{eV}$ energy would be gained by a decay to the unoccupied metal states above $E_{\mathrm{F}}$. We note that long-living electrons are found for all coverages above $1 \mathrm{BL} \mathrm{D}_{2} \mathrm{O} / \mathrm{Ru}(001)$, which forms a $\sqrt{3} \times \sqrt{3}$ ordered wetting layer. At higher coverage crystalline $\mathrm{D}_{2} \mathrm{O}$ clusters are formed on top of the wetting layer $(37,38)$. Assuming coherent tunneling of a quasi-free electron through a rectangular $1 \mathrm{eV}$ barrier and a cluster thickness $d=1.9 \mathrm{~nm}$ (39) for the average coverage of $2 \mathrm{BL}$ used in Fig. 1 provides decay times on the order of $\mu$ s. Thus, tunneling with a barrier height defined by the IPS cannot explain our observation even when taking into account the thickness added by crystalline ice clusters. At the heart of the phenomenon lies the hydration process that dresses the electron with rearranged adjacent water molecules as evidenced by the time-dependent energy shift and that must effectively shield the electron from the metal surface. This enhances the barrier height and significantly reduces the tunneling probability. 
A microscopic description of the observed phenomenon would benefit from understanding whether the trapped electrons $\mathrm{e}_{\mathrm{T}}$ reside at the ice surface or in the ice bulk. To answer this question, the solvation site is probed by a Xe overlayer experiment following Refs. 40,41. Adsorption of $\mathrm{Xe}$ forms an adlayer on top of the ice structure, which modifies the environment of the excess electrons and thus their spectroscopic signature if these electrons reside at the surface (41). Fig. 5 demonstrates that the $\mathrm{e}_{\mathrm{T}}$ peak is quenched upon $\mathrm{Xe}$ adsorption (dotted line). This observation unambiguously shows that the trapping sites that accept $\mathrm{e}_{\mathrm{T}}$ electrons reside at the ice-vacuum interface. Recovery of the $\mathrm{e}_{\mathrm{T}}$ peak after thermal desorption of $\mathrm{Xe}$ - whereby the ice remains adsorbed - evidences that $\mathrm{e}_{\mathrm{T}}$ sites have not been destroyed. This scenario is supported by our first-principles calculations shown below in Sec. 5: Pre-existing defects are responsible for trapping the electron and triggering its subsequent dynamical (dipole-induced) stabilization.

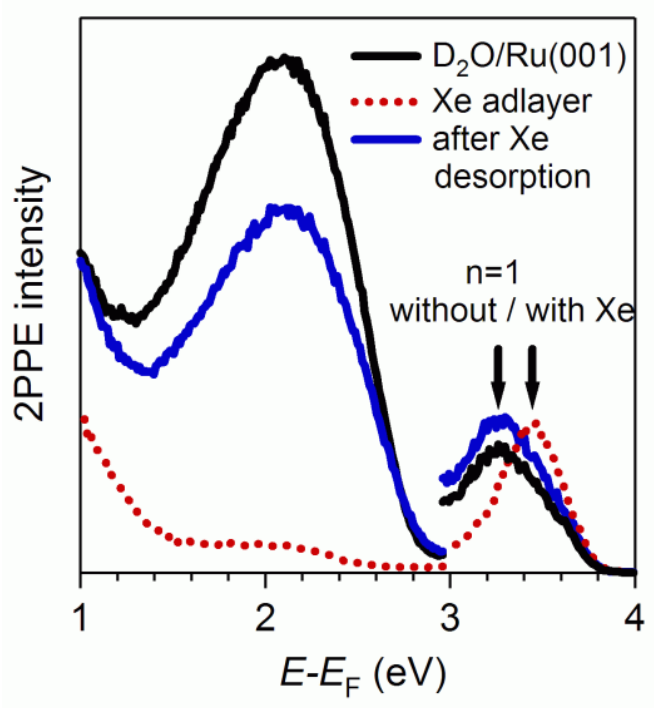

Fig. 5: Single color 2PPE spectra taken at $40 \mathrm{~K}$ before and after $\mathrm{Xe}$ adsorption on $\mathrm{D}_{2} \mathrm{O} / \mathrm{Ru}(001)$. The $\mathrm{e}_{\mathrm{T}}$ peak is quenched upon $\mathrm{Xe}$ adsorption, which is direct evidence for the residence of $\mathrm{e}_{\mathrm{T}}$ electrons at the ice-vacuum interface. Upon Xe desorption at $85 \mathrm{~K}$ - the ice remains adsorbed at this temperature - the $\mathrm{e}_{\mathrm{T}}$ peak recovers. The shift in the $n=1$ IPS binding energy (solid arrows) upon Xe adsorption quantifies the respective work function change which can not explain the quenching of the $\mathrm{e}_{\mathrm{T}}$ state. Intensities are strongly temperature-dependent near $40 \mathrm{~K}$ and are normalized to the low-energy cutoff at $E-E_{\mathrm{F}}=0.4 \mathrm{eV}$. 


\section{Thermally activated relaxation among conformational substates}

We now discuss the time-dependent evolution of the binding energy and the population decay, which has been observed in the fs to ps range at amorphous ice-metal interfaces $(24,34,40)$ and in liquid water $(42)$, where it has been attributed to dynamical screening during the solvated electron formation. However, for crystalline ice on $\mathrm{Ru}(001)$, we observe a localized state $\left(\mathrm{e}_{\mathrm{T}}\right)$ stabilized by $1 \mathrm{eV}$ with respect to the delocalized state $(n=1)$ (Fig. 3 ). The population decay is analyzed by the time-dependent $2 \mathrm{PPE}$ intensity of the $\mathrm{e}_{\mathrm{T}}$-feature. It is non-exponential as seen from the inset in Fig. 6a which is the consequence of the dynamically varying wave function overlap between donor and acceptor states. We find that the population decay in crystalline ice is slowed down by 14 orders of magnitude compared to amorphous ice $(24,27)$ and by 11 orders compared to liquid water $(8)$. The stabilization in crystalline ice is manifested by a shift in binding energy of $0.6 \mathrm{eV}$ within $10 \mathrm{~min}$ (Fig. 6a) compared to $0.3 \mathrm{eV}$ within $1 \mathrm{ps}$ in amorphous ice (24). Thus we conclude that in crystalline ice and amorphous structures on metal substrates at typical $\mathrm{D}_{2} \mathrm{O}$ coverage of 2-10 BL the localized electronic states and their dynamics are fundamentally different.
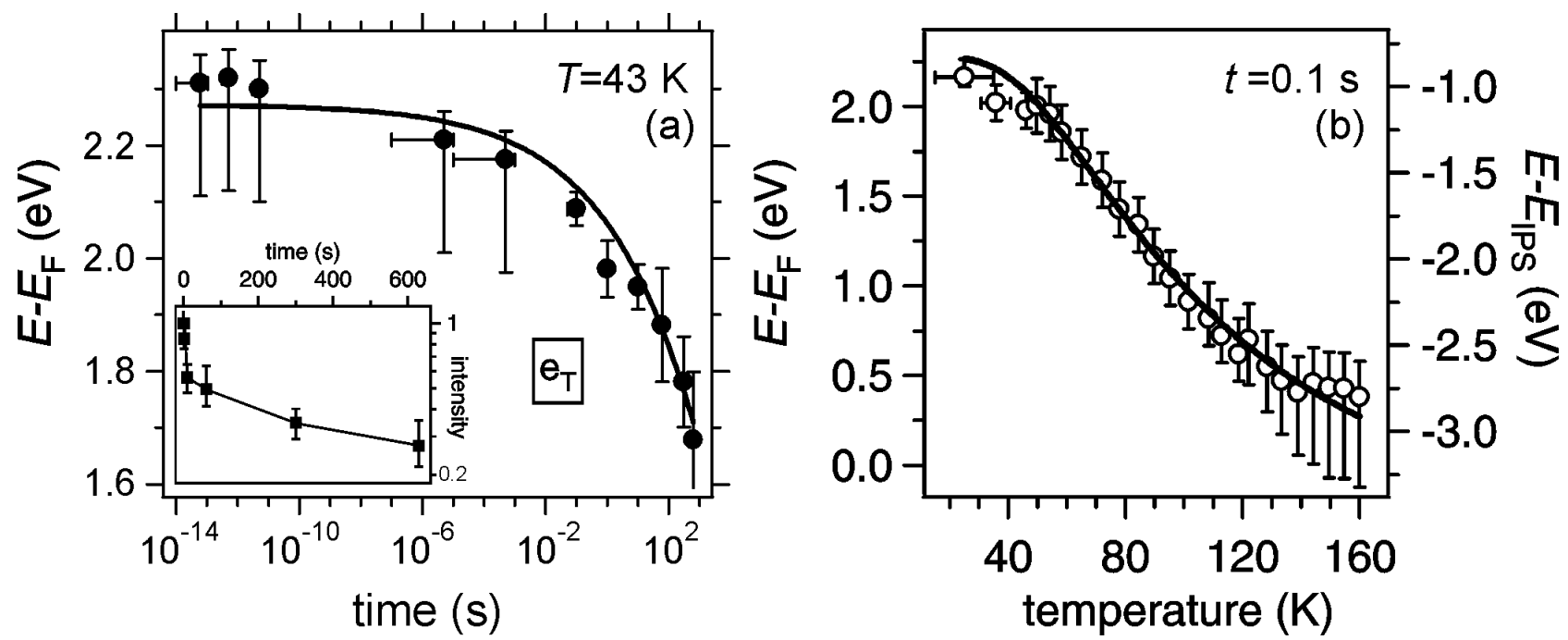

Fig. 6: (a) The temporal evolution of $\mathrm{e}_{\mathrm{T}}$ binding energies is depicted as a function of time over 17 orders of magnitude in time. The solid line is based on dynamical stabilization among conformational substates described by a harmonic potential as a function of the multidimensional nuclear coordinate $q$. The inset presents the $\mathrm{e}_{\mathrm{T}}$ 
intensity integrated over the peak on a logarithmic scale. In (b) the temperature-dependent stabilization energy of the $\mathrm{e}_{\mathrm{T}}$ peak for crystalline $\mathrm{D}_{2} \mathrm{O}$ on $\mathrm{Ru}(001)$ at a fixed delay of $\sim 0.1 \mathrm{~s}$ after excess electron generation is given. Left axis: energy with respect to $E_{\mathrm{F}}$; right axis: stabilization with respect to the $n=1$ IPS. The solid line results from a simultaneous description of temperature- and time-dependent data considering dynamical stabilization among conformational substates.

The slow stabilization and decay can be described in terms of a continuous nuclear reorganization $(13,43,44)$ during which the electron relaxes deeper into the trap passing through adjacent conformational substates. These substates characterize local minima on the potential energy surface separated by energy barriers $E_{\mathrm{a}}$. Dispersive transport or relaxation among isoenergetic conformations are known to be empirically well described by a stretched exponential law $(16,17,45,46)$. Here, we apply this concept to describe the energy gain of the excess electron:

$$
E(t, T)=A \cdot \exp \left[-2 \cdot(t / \tau)^{\beta} \cdot \exp \left(-E_{\mathrm{a}} / k_{\mathrm{B}} T\right)^{\beta}\right]
$$

This expression reproduces our observation with $E_{\mathrm{a}}=95(5) \mathrm{meV}, \beta=0.16(3)$, and a relaxation time $\tau=4(1) \mathrm{ms}$ (solid line in Fig. 6a). The relaxation time, which is long compared to typical vibrational frequencies, suggests that correlation among the individual microscopic processes plays a relevant role for the screening of the excess electron. The activated evolution through the different conformational substates characterizes the stabilization of the excess electron by molecular reorganization, and $E_{\mathrm{a}}$ estimates the average barrier between the substates. This description also holds for the temperature dependence. Fig. $6 \mathrm{~b}$ depicts the binding energies for the $\mathrm{e}_{\mathrm{T}}$ state at constant time delay and ramped temperature. The energy gain with respect to the $n=1$ IPS is increased for larger $T$ and reaches $3 \mathrm{eV}$ at $160 \mathrm{~K}$, as temperature supports faster stabilization by thermal activation to overcome the barriers between the conformational substates. However, although the stretched exponential describes the observed stabilization dynamics extremely well from femtoseconds to minutes, it does not offer insight into the corresponding molecular rearrangements. 


\section{Ab initio description of initial trapping sites}

Microscopic insight into the initial trapping sites is gained by ab initio calculations based on first-principles density functional theory. Generic defect structures are modeled on the (0001)-surface of hexagonal ice $\left(I_{h}\right)$, a model that is frequently employed in theoretical investigations $(10,47,48,49)$. In the absence of detailed knowledge of the morphology of the crystalline ice clusters this computationally still tractable model surface gave access to the properties of fundamental surface defects and their ability to bind an excess electron. Since the important features of interest here are inherently localized to the surface bi-layer, we expect that the illustrative results obtained with this model are transferable to the structurally related (111) surface of the metastable cubic ice $\left(I_{c}\right)$. Cubic ice is expected to form first during the deposition of water under the experimentetal conditions. The calculations were performed with a plane wave supercell method utilizing norm conserving Troullier-Martins pseudopotentials and a basis with plane wave up to a kinetic energy of $80 \mathrm{Ry}$ as described in detail in Ref. 10. The BLYP exchange-correlation energy functional was used for the neutral structures. In the presence of the excess electron an additional approximate correction for the self-interaction was employed as described and tested in Ref. 10. The approach we pursue here allows for an interaction of the excess electron with the valence electrons of individual water molecules and hence gives a realistic account of the decay of the excess electron state at a surface trap into the ice layer. DFT methods utilizing gradient corrected functionals like BLYP-functional successfully described water systems (10), yet from extensive benchmarking (50) of neutral water cluster the BLYP functional is known to underestimate the binding energy of water molecules by $35 \mathrm{meV}$ compared to more sophisticated methods like MP2 or X3LYP. Such an error is clearly acceptable for the present illustrative analysis of trapping sites and their formation energies in the neutral state. The binding energy of the excess electron to the trapping site is evaluated with respect to the configuration without the trapping site $(51)$. 
The surface traps are represented in a $4 \times 4$ surface cell of $I_{h}(0001)$ of 3 bi-layers of ice and a vacuum region corresponding to 5 bi-layers (cross checked with 10 bi-layers of vacuum). For the investigation of the excess electron binding to the traps, a larger vacuum of 10 bi-layers was consistently used for both neutral and charged trap configurations. Only by this large surface unit cell we are able to localize the electron laterally at the traps. With this simple model surface we investigated surface vacancies (an entirely missing $\mathrm{H}_{2} \mathrm{O}$-molecule), divacancies, and orientational defects in the first bi-layer and admolecules to the surface bilayer. Each of these generic defects is expected to be present on the surface of the crystalline ice clusters albeit with different abundance.

The geometry of the ice surface and exemplary orientational defects created during the preparation of the ice surface of water molecules with orientations $\mathrm{S}_{\mathrm{A}}$ and $\mathrm{S}_{1}$ (defined below) are shown in Fig. 7a. The water molecules $S_{2}$ (subsurface layer) and $S_{A}$ (surface layer) both accept hydrogen bonds from adjacent bi-layers or water molecules adsorbed on the surface and the molecules with orientations $S_{D}$ (surface layer) and $S_{1}$ (sub surface layer) donate hydrogen bonds. For the orientational defects of Fig. 7a and the other defects we determine the respective formation energies $E_{\mathrm{d}}(10)$ as a measure for their relevance. Note that in thermal equilibrium the formation energy determines the abundance of the surface defects. For the surface vacancies of water molecules with all four orientations and a di-vacancy, the calculated formation energies were too high to explain the abundance of the observed traping sites at the given temperature and these are not treated in detail here. However, a strong electron trap was not found among these vacancies. The most favorable orientational defect $\left(E_{\mathrm{d}}=0.25 \mathrm{eV}\right)$ is a re-oriented water molecule $\mathrm{S}_{\mathrm{A}}$ such that one OD-bond points towards the vacuum. The molecule assumes a $\mathrm{S}_{\mathrm{D}}$-configuration and a Bjerrum L-defect is created in between the $S_{A}$ and the neighboring molecule (here a $S_{2}$-molecule), accompanied by a sideways reconstruction of the under-coordinated $\mathrm{D}_{2} \mathrm{O}$ neighbor $\mathrm{S}_{2}$ (see Fig. 7c). Besides the 
presence of the Bjerrum L-defect at the surface the reoriented $S_{A}$ molecule in this case is surrounded by four $S_{D}-$ molecules. We refer to this configuration in the following as $S_{A}$-flip. In contrast to a re-orientation of water molecules in the bulk, a Bjerrum D-defect does not occur here due to the proximity to the surface. The molecule $S_{1}$ of the subsurface layer connects the topmost bi-layer to the bulk. Its reorientation gives rise to a configuration we label $\mathrm{S}_{1}$-flip $\left(E_{\mathrm{d}}\left(\mathrm{S}_{1}\right.\right.$-flip$\left.)=0.45 \mathrm{eV}\right)$ and results in an undercoordination of this and the watermolecule in the adjacent bi-layer (Bjerrum L-defect). By a propagation of these Ldefects to a neighboring $\mathrm{S}_{\mathrm{D}}$-water molecule the natural coordination of surface and subsurface water molecules can be restored as illustrated for $S_{A^{-}}$flip in Fig. $7 d$. The process requires the reorientation of two OD-bonds and converts the orginal $S_{A}$ and $S_{D}$ molecules into those of the opposite kinds $S_{D}$ and $S_{A}$ respectively. Note, that the newly formed $S_{D}$ (derived from $\mathrm{S}_{\mathrm{A}^{-}}$flip) unlike the other $\mathrm{S}_{\mathrm{D}}$ of our initial configuration has three nearest $\mathrm{S}_{\mathrm{D}^{-}}$ neighbors. This structure, we refer to as $\mathrm{S}_{\mathrm{AD}}$, can be understood in terms of the proton disorder. Compared to the initial configuration the $\mathrm{S}_{\mathrm{AD}}$ structure has a formation energy of only $60 \mathrm{meV}$. $\mathrm{S}_{\mathrm{AD}}$ should thus be an abundant structure at the ice surface. We also considered a reorientation of $\mathrm{S}_{\mathrm{D}}$ with two hydrogen located in vacuum resembling the binding site in clusters (52) that turned out to be unstable even in the presence of the excess electron. Although such a defect can be stabilized by a reconstruction similar to $\mathrm{S}_{\mathrm{AD}}$, it is not relevant as an initial trap due to its high formation energy. Excess electrons bind to such orientational defects or surface-water configurations similar to $\mathrm{S}_{\mathrm{AD}}$ in a laterally well localized state, as shown for $\mathrm{S}_{\mathrm{A}}$-flip and $\mathrm{S}_{\mathrm{AD}}$ in Fig. 7c,d. The electron density essentially resides outside the surface and decreases by six orders of magnitude from its maximum values to the third bi-layer. This is demonstrated in Fig. 7e where we show the in-plane average of the square modulus of the excess electron's wave function $\left\langle|\Phi|^{2}\right\rangle_{x, y}$, at $S_{A^{-}}$flip vs. the distance from the normal to the surface $z$. Due to this pronounced decrease, the tunneling 
probability will be sufficiently reduced until subsequent reorganization promotes further vertical localization.
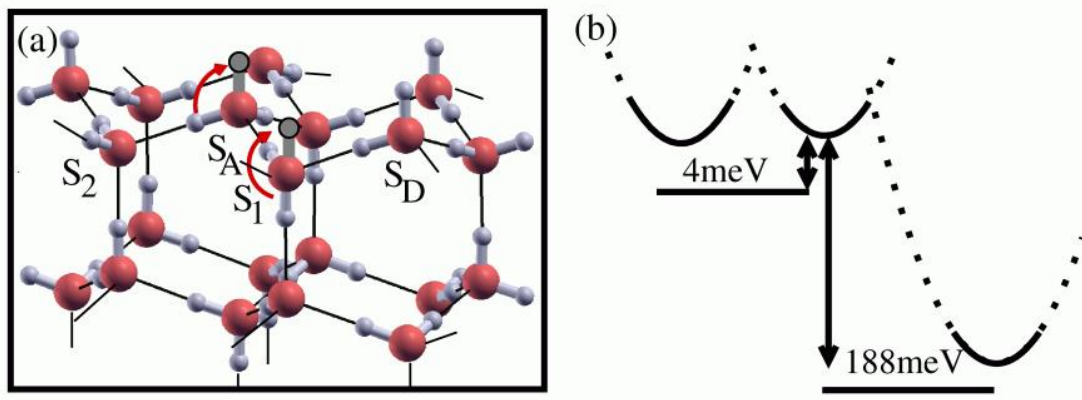

(e) $\left.<|\Phi|^{2}\right\rangle_{x, y}$ (arb. units)
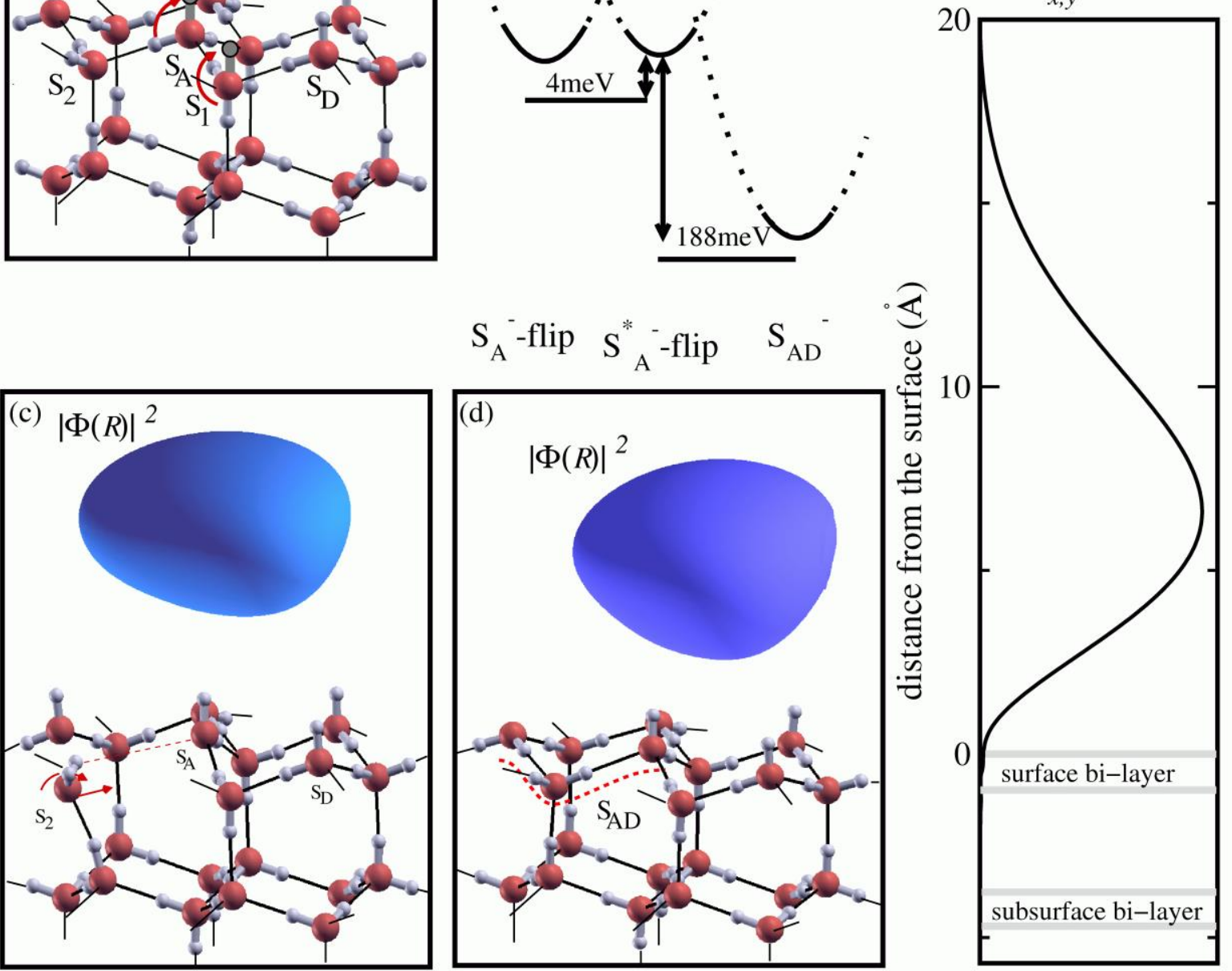

Fig. 7: Orientational defects: (a) formation of $S_{A}$-flip and $S_{1}$-flip on the (0001)-ice surface, the rearrangement of the $\mathrm{D}_{2} \mathrm{O}$ molecules $\mathrm{S}_{\mathrm{A}}$ and $\mathrm{S}_{1}$ is indicated by arrows (curved arrows: rotations of molecules about an O-D axis; straight arrow: molecule displacement). Only part of the surface unit cell is shown and the continuation of the water network is indicated. (b) Energies of $\mathrm{S}_{\mathrm{A}}-\mathrm{flip}, \mathrm{S}_{\mathrm{AD}}$, and the intermediate configuration $\mathrm{S}_{\mathrm{A}}{ }^{*}$-flip as described in the text. (c) Geometry of $\mathrm{S}_{\mathrm{A}}$-flip and the electron density of the excess electron $|\Phi(R)|^{2}$ at $70 \%$ of its maximum value. The electron is bound to both DO-bonds at the neighbouring $S_{A}$ and $S_{D}$. Note the large movement of $S_{A}$ and its neighbour away from the ideal sites. The reconstruction of the bond network to form $\mathrm{S}_{\mathrm{AD}}$ is indicated by arrows (see above) (d) Geometry of $\mathrm{S}_{\mathrm{AD}}$ and the excess electron density $|\Phi(R)|^{2}$ as for $\mathrm{S}_{\mathrm{A}}$-flip; the dashed line indicates new hydrogen bonds and the involved sites. (e) In-plane average of the density of the excess electron vs. the distance from the surface at $S_{A}$-flip to illustrate its negligible overlap with the ice/metal system (similarly for the $\mathrm{S}_{\mathrm{AD}}$ defect). The gray horizontal lines indicate the position of the oxygen layers.

Upon binding to the orientational defects the excess electron typically gains energy of more than $350 \mathrm{meV}$. Only $60 \mathrm{meV}$ of this energy gain arise from local structural relaxation. Thus, 
the observed energy relaxation of more than $0.5 \mathrm{eV}$ must involve a complex reconstruction process through many conformational states as illustrated by the energy diagram in Fig. $7 \mathrm{~b}$. Of course on the surface some structures with a larger binding energy for excess electrons may pre-exist at low concentration which later on evolve in a similar manner. Such vacancies would naturally explain the width of the initial spectra. As exemplified here for the $S_{A}$-flip, the rupture of the water network by the creation of orientational defect and the simultaneous formation of a Bjerrum L-defect may give rise to reconstructions that restore the hydrogen bond network or correspondingly the diffusion of the L-defect to other surface sites where it can be annihilated by the reorientation of a surface water molecule. Such steps are indicated by red arrows in Fig. $7 \mathrm{c}$ for the neighbours $S_{2}$ and $S_{D}$ which yield the $S_{A D}$-structure. By gyrating $\mathrm{S}_{2}$, the Bjerrum L-defect migrates away from its original site and the $\mathrm{S}_{\mathrm{A}}$-site retains its three-fold coordination in the intermediate configuration $\mathrm{S}_{\mathrm{A}}{ }^{-}$flip. The hydrogen bond between $S_{2}$ and $S_{D}$ is restored by converting the free OD of $S_{D}$ into a $S_{A}$-like orientation and the defect $S_{A}$-flip into a molecule of type $S_{D}$. By this conversion the excess electron gains $0.19 \mathrm{eV}$. In the presence of the excess electron, $\mathrm{S}_{\mathrm{AD}}$ even has a negative formation energy and its dynamical creation would stabilize the electron-ice complex at the surface. The above migration of surface Bjerrum L-defects also facilitates the motion of a $\mathrm{S}_{\mathrm{D}}$-type orientation to a $\mathrm{S}_{\mathrm{A}}$-site via dynamic formation of a $\mathrm{S}_{\mathrm{A}}$-flip.

Also admolecules are abundant trapping sites for the excess electron at the surface. Theoretical work $(47,48)$ predicted high adsorption energies for admolecules on the $I_{h}(0001)$ that compares or even exceeds the cohesion energy of the bulk, a phenomenon could give rise to rough surfaces (48). Among four prototypical binding configurations (47), the admolecule most favorably interacts with two neighboring $S_{D}$ and one $S_{A}$ surface water molecules arranged in a triangle as sketched in Fig. 8 or similarly with two $S_{A}$ and one $S_{D}$. Only in the former case the admolecule adsorbs with one OD-bond pointing into the vacuum. Here we investigated such a configuration as a trapping site for the excess electron. As 
expected from previous DFT-work [48], where the adsorption energy was found to be slightly lower than the cohesive energy, we find that the admolecule shown in Fig. 8 has a low formation energy of $130 \mathrm{meV}$. For the other configuration not shown here we find slightly lower formation energy of $90 \mathrm{meV}$. The excess electron density (cf. Fig. 8b) is laterally well localized and decays by five orders of magnitude from its maximum value to the third bi-layer. This is one order of magnitude less than in the previous case of the orientational defects and the $\mathrm{S}_{\mathrm{AD}}$. Also the lateral localization of the excess electron at the admolecule is somewhat weaker than in case of $\mathrm{S}_{\mathrm{A}}-\mathrm{flip}$ and $\mathrm{S}_{\mathrm{AD}}$. Batista and Jonssón showed (47) that the neutral admolecules are mobile entities and predicted diffusion barriers of about $0.2 \mathrm{eV}$. An aggregation of admolecules at an initial trapping site thus also would contribute to the effective screening of the excess electron. 


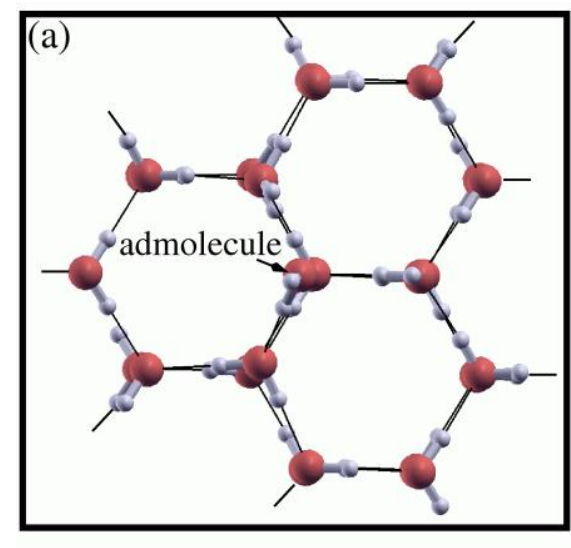

(c)
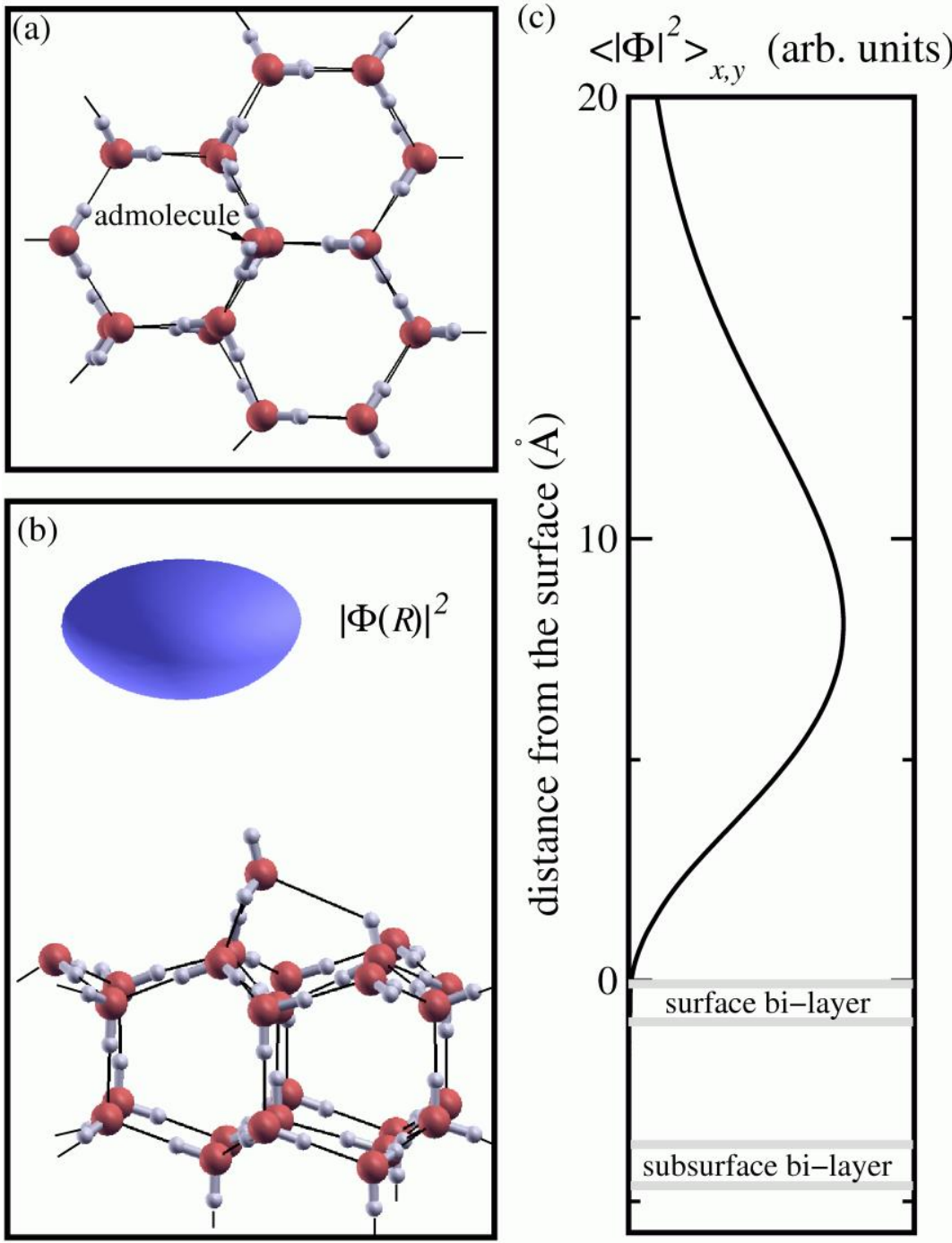

Fig. 8: Admolecule: (a) geometry of an admolecule absorbed (top-view) and (b) side view together with the electron density of the excess electron $|\Phi(R)|^{2}$ at $70 \%$ of its maximum value. Only a part of the surface unit cell is shown and the continuation of the water network is indicated. (c) In-plane average of the density of the excess electron vs. the distance from the surface. The admolecule is located $2.09 \AA$ above the surface layer $(\mathrm{O}-\mathrm{O}$ distance). Note, the weaker localization in comparison to the $\mathrm{S}_{\mathrm{AD}}$-structure.

\section{Discussion}

\subsection{Comparison of experiment and theory}

Our $a b$ initio calculations demonstrate that excess electrons may populate reorientational defects and related structures that originate from proton-disorder or admolecules at crystalline ice surfaces and which result in considerable binding energy gain of the excess electrons. We turn now to a discussion that links the theoretical and experimental findings. Similar to the aggregation of dipole moments at the initial binding site concluded in the 
previous section, the reconstruction of the $S_{1}$-flip or of a variant of the $S_{A}$-flip goes along with the diffusion of the associated Bjerrum L-defect into subsurface layers. This process directs the initially disordered (under the auspice of the ice-rules) water dipole moments towards the excess electron. Once the excess electron is trapped the dynamical creation of orientational defects or other defects can be envisaged. For example a dynamical reorientation of $\mathrm{S}_{1}$-molecules (10), of $\mathrm{S}_{\mathrm{A}}$-molecules (as described above), or of $\mathrm{S}_{\mathrm{D}}$ such that both $\mathrm{D}$ atoms are directed towards the excess electron is conceivable, as the corresponding potential energy surface is quite flat. Such a structural evolution of the defects via conformational substates separated by barriers is thermally activated and hence accelerated at increased temperatures in agreement with the experimental observation (see Fig. 6b). It enhances not only the local dipole moment and hence the electron's binding energy, but also its localization. Recent mixed quantum-classical molecular dynamics simulations (53) of the stabilization of excess electrons at the ice/air interface at $200 \mathrm{~K}$ support our conclusions regarding the reorganization of the hydrogen bond network. There, the energy gain occurs while the ice surface passes through a large number of such reorganization steps by which the electron enters a larger void formed in the disordered liquid-like surface bi-layer at that temperature. In such a scenario water molecules are removed from surface and subsurface layers and form admolecules on the surfaces. Thereby the proper orientation of dangling ODs in the void and of the admolecule aids the binding and screening of the excess electron. Thus reorganization processes via conformational substates as described above is of relevance here, too.

As we have shown, the initial trapping of the excess electron at surface defects, local protondisorder structures, and admolecules is a mandatory requisite for the initiation of the reorganization process and for the explanation of the long-living state. These traps could be created dynamically as described in Refs. 10, 53. In our work, however, the experimental observation of a population via the very shortlived IPS (see Fig. 3) strongly suggest the 
trapping sites are already present at the surface and originate from the growth process of the ice. These pre-existing traps prevent the excess electron from recombining with the metal before further reorganization induced screening takes place. Furthermore, the trap hinders the electron to diffuse from the ice cluster surface to the wetting layer where fast relaxation occurs.

Trapping sites microscopically similar to the ones described here could exist on an amorphous ice layer as well. Thus in principle similar mechanisms as we discussed here for the long living excess electron state may also support such long living electron states on amorphous ice/metal systems. Amorphous ice is characterized by a strained disordered water network (54) with a larger concentration of under-coordinated water molecules and surface defects. According to our experience the isolated character of the described trapping sites with smaller supercells is a requisite for a strong lateral localization of the trapped electron. However, on the amorphous ice the excess electron may interact with more than one surface defect, which should results in a rather delocalized excess electron state. As a consequence it may remain sufficiently mobile to migrate and transfer back to the metal via sites where efficient recombination channels exist. Such a scenario most likely leads to the population decay on ps timescales observed for amorphous ice and inhibits further stabilization on timescales of minutes. In other words, the fact that the density of pre-existing traps in crystalline ice is orders of magnitude smaller prevents the excess electron from migrating on the ice surface to recombination channels with the metal, and thus aiding to set-up further reconstructions that increase the local dipole. This leads to further (lateral and vertical) localization outside the ice surface, and a continuous increase in the binding energy. Clearly such dipolar reorganization is likely to take place on time scales of up to minutes supporting the stretched exponential model discussed above. Future experiments on thicker amorphous ice layers could facilitate an experimental determination of the concentration ratio of long living trapping sites for amorphous and crystalline ice structures. Considering the 
understanding developed in the present study, a long-living state could appear also for a sufficiently thick amorphous ice layer on a metal substrate. The large distance set by the ice thickness to prevent ultrafast electron transfer to the metal is considered to enhance the time window available for experimental observation of the long-living feature. Our control experiments showed that up to $10 \mathrm{BL}$ thick amorphous films do not present a feature comparable to $\mathrm{e}_{\mathrm{T}}$ found for crystalline ice. If successful for larger thickness, a detailed thickness dependent 2PPE study could actually lead to a determination of the average diffusion rate and velocity. However, this is out of the scope of the present article.

\subsection{Impact of the ice structure on $e_{T}$ population and decay}

The key observation of our work is the observation of extremely long-living electrons $(>2 \mathrm{eV}$ above $\left.E_{\mathrm{F}}\right)$ at $\mathrm{nm}$ distance in front of the metal surface. Due to cluster formation of the crystalline $\mathrm{D}_{2} \mathrm{O}$ ice on top of the first wetting bilayer on $\mathrm{Ru}(001)$ one might consider that a simple thickness dependence of the tunneling barrier to be responsible for the observed lifetimes up to minutes. However, such a scenario is in disagreement with the experimental observations due to several reasons given below.

Analysis of the 2PPE intensity of the $n=1$ IPS yields according to Ref. 39 for 2 BL mass equivalent coverage an average crystalline ice cluster thickness of 4 BL. Including the ordered first bilayer the distance from the ice-metal interface to the top of the crystalline ice islands

amounts to $5 \mathrm{BL}$ or $19 \AA$ A. As discussed in Sec. 3 lifetimes on the order of $10^{-6} \mathrm{~s}$ are expected for a reasonable cluster thickness. Unrealistically thick clusters are required to arrive at the observed lifetimes up to minutes.

As we show in Sec. 3 and Fig. 3, injection of the excess electrons proceeds through the $n=1$ IPS. As the IPS intensity in 2PPE increases with decreasing coverage down to $1 \mathrm{BL}$ (not shown) the IPS wave function is confined to lateral regions of the bare first BL considering the islands structure of crystalline ice $\mathrm{D}_{2} \mathrm{O} / \mathrm{Ru}(001)$. The $n=1$ IPS wave function extends from 
the first BL into vacuum typically up to $20 \AA$. To populate the long-living electrons the latter must reside at distances to the metal surface up $20 \AA$ which represents an upper limit for the distance of the excess electron to the metal. Note, that this estimate is in agreement with the cluster thickness estimated in the previous paragraph. For thicker clusters the wave function overlap of the IPS with the trapping site at the cluster surface disappears which would inhibit any long living electron signature in 2PPE. Our DFT calculations actually show that the orientational defects explaining the long-living electrons adjacent to the ice-metal interface do exist at the crystalline ice surface and exhibit reasonable formation energies and negligible coupling to the metal. More generic defects like surface vacancies have been reported to show a binding energy gain up to $1 \mathrm{eV}$ [49], however the additional dynamic evolution of the binding energy observed in the present study is not included in this study.

The timescales related to decay by coherent tunneling through a smooth molecular adlayer of a controlled nm thick molecular layer have recently been determined for amorphous ammonia layers on a $\mathrm{Cu}(111)$ substrate (55). The observed decay times are all faster than $500 \mathrm{ps}$ - far too fast to explain residence times of minutes. Extrapolation of the thickness dependent decay times in amorphous ammonia layers to values reported for crystalline ice structures results in a distance of $16 \mathrm{~nm}$ between the excess electron and the metal. Considering that we observe the long living state in crystalline ice $\mathrm{D}_{2} \mathrm{O} / \mathrm{Ru}(001)$ already for the very small coverage of only 1.2 BL, whereby $1 \mathrm{BL}$ forms the ordered wetting layer, formation of $16 \mathrm{~nm}$ high islands from a mass equivalent of $0.2 \mathrm{BL}$ is due to thermodynamic reasons unphysical.

We therefore conclude that the cluster like morphology of crystalline ice structures on top of a ordered wetting layer cannot explain the long-living electrons. Essential to understand the residence times up to minutes in combination with the ongoing stabilization is the formation of an water-electron complex that through molecular rearrangement screens the electron from the metal substrate. 


\section{Outlook and Conclusion}

In summary, we have shown that excess electrons localize at surface defects of crystalline ice and stabilize dynamically by transitions between different conformations. These excess electrons initially bind to pre-existing surface traps, which prevent decay to a metal substrate even at $\mathrm{nm}$ distance. Subsequently the electrons are well screened by molecular rearrangement. Stabilization evolves from femtoseconds to minutes, i.e. from the ultrafast electronic excitations to quasi-equilibrium, and is unveiled by a continuous binding energy increase of the excess electron over 17 orders of magnitude in time. Such long-living excited electronic states have broad impact on various fields. Kim et al. concluded from life time measurements using $2 \mathrm{PPE}$ an increase in photoinduced dissociative electron attachment for $\mathrm{CFCl}_{3}$ adsorbed on amorphous ice films [56]. For crystalline ice the charge density and the long residence time of excess electrons in front of the surface should further enhance the reactivity and indeed in experiments using oxygen or chlorofluorocarbons as a reactant we find an extremely efficient dissociative electron attachment [57]. Thus, our findings might be relevant for astrophysics or atmospheric chemistry. Furthermore, long-living electronic states are a prerequisite for efficient molecular switches in molecular electronic devices. Also, trapped charges in water are crucial in fuel cells and insight into the evolution of excited electronic states might further their optimization. 


\section{Acknowledgement}

We are grateful for experimental support by M. Bertin and acknowledge funding by the Deutsche Forschungsgemeinschaft through SPP 1093, Sfb 450 and 658, by the GermanIsraeli Foundation, by the European Community through NoE Nanoquanta (NMP4-CT2004-500198), SANES (NMP4-CT-2006-017310), Spanish MEC (FIS2007-65702-C02-01), and NANO-ERA Chemistry projects, UPV/EHU (SGIker Arina). The authors thankfully acknowledge the computer resources, technical expertise and assistance provided by the Barcelona Supercomputing Center-Centro Nacional de Supercomputación.

SYNOPSIS TOC

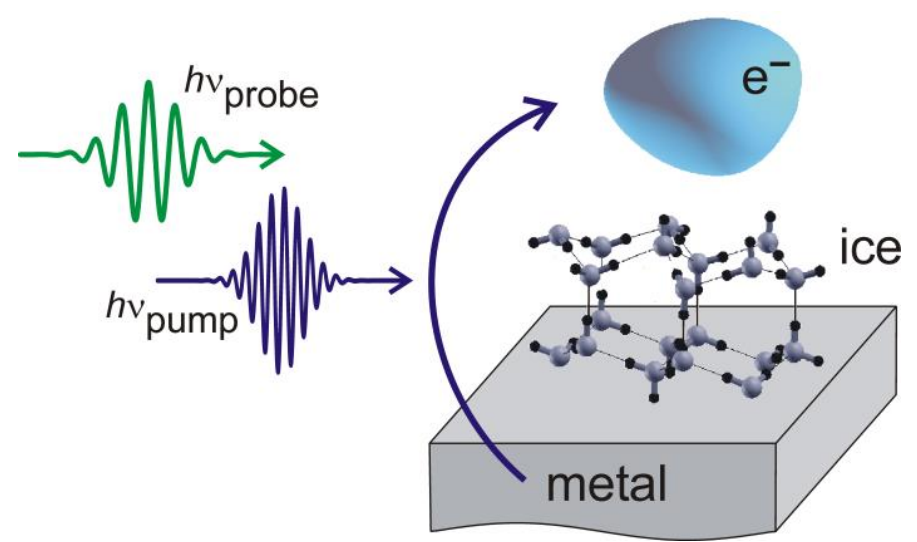


${ }^{1}$ Lu Q.-B. and Sanche L. Phys. Rev. Lett. 2001, 87, 078501.

${ }^{2}$ Verlet J. R. R., Bragg A. E., Kammrath A., Cheshnovsky O., Neumark D. M. Science 2005, 307, 93.

${ }^{3}$ Turi L., Sheu W.-S., Rossky P. J. Science 2005, 309, 914.

${ }^{4}$ Lin J., Balabin I. A., Beratan D. N. Science 2005, 310, 1311.

${ }^{5}$ Sieger M. T., Sieger W. C., Orlando T. M. Nature 1998, 394, 554.

${ }^{6}$ Garrett B. C. et al. Chem. Rev. 2005, 105, 355.

${ }^{7}$ Kevan L. Acc. Chem. Res. 1981, 14, 138.

${ }^{8}$ Madsen D., Thomsen C. L., Tøgersen J., Keiding S. R. J. Chem. Phys. 2000, 113, 1126.

${ }^{9}$ Barnett R. N., Landman U., Cleveland C. L., Jortner J. Phys. Rev. Lett. 1987,59, 811.

${ }^{10}$ Baletto F., Cavazzoni C., Scandolo S., Phys. Rev. Lett. 2005, 95, 176801.

${ }^{11}$ Lehr L., Zanni M. T., Frischkorn C., Weinkauf R., Neumark D. M. Science 1999, 284, 635.

${ }^{12}$ Zhu X.-Y. J. Phys. Chem. B 2004, 108, 8778.

${ }^{13}$ Miller R. J. D., McLendon G. L., Nozik A. J., Schmickler W., Willig F.; Surface Electron Transfer Processes, VCH, New York, 1995.

${ }^{14}$ Levy Y. and Onuchic J. N. Annu. Rev. Biophys. Biomolec. Struct. 2006, 35, 389.

${ }^{15}$ Frauenfelder H., Parak F., Young R. D. Ann. Rev. Biophys. Biophys. Chem. 1988, 17, 451.

${ }^{16}$ Frauenfelder H., Fenimore P. W., McMahon B. H. Biophys. Chem. 2002, 98, 35.

${ }^{17}$ Ansari A., Jones C. M., Henry E. R., Hofrichter J., Eaton W. A. Science 1992, 256, 1796.

${ }^{18}$ Ansari A., Kuznetsov S. V., Shen Y. PNAS 2001, 98, 7771.

${ }^{19}$ Jäger M., Nguyen H., Crane J. C., Kelly J. W., Gruebele M J. Mol. Biol. 2001, 311, 373.

${ }^{20}$ Caliskan G., Mechtani D., Roh J. H., Kisliuk A., Sokolov A. P., Azzam S., Cicerone M. T., Lin-Gibson S., Peral I. J. Chem. Phys. 2004, 121, 1978.

${ }^{21}$ Echenique P. M., Berndt R., Chulkov E.V., Fauster Th., Goldmann A., Höfer U. Surf. Sci. Rep. 2004, 52, 219.

${ }^{22}$ Föhlisch A., Feulner P., Hennies F., Fink A., Menzel D., Sanchez-Portal A., Echenique P. M., Wurth W. Nature 2005, 436, 373.

${ }^{23}$ Ge N.-H., Wong C. M., Lingle Jr. R. L., McNeill J. D., Gaffney K. J., Harris C. B. Science 1998, 279, 202.

${ }^{24}$ Gahl C., Bovensiepen U., Frischkorn C., Wolf M. Phys. Rev. Lett. 2002, 89, 107402.

${ }^{25}$ Miller A. D., Bezel I., Gaffney K. J., Garrett-Roe S., Liu S. H., Szymanski P., Harris C. B. Science 2002, 297, 1163.

${ }^{26}$ Li B., Zhao J., Onda K., Jordan K. D., Yang J., Petek H. Science 2006, 311, 1436.

${ }^{27}$ Stähler J., Gahl C., Bovensiepen U., Wolf M. J. Phys. Chem. B 2006, 110, 9637.

${ }^{28}$ Piotrowiak P., Galoppini E., Wei Q., Meyer G. J., Wiewor P J. Am. Chem. Soc. 2003, 125, 5278.

${ }^{29}$ Zhao J., Li B., Onda K., Feng M., Petek H., Chem. Rev. 2006, 106, 4402.

${ }^{30}$ Smith R. S. and Kay B. D. Surf. Rev. Lett. 1997, 4, 781.

${ }^{31}$ The term bilayer refers to the rugged structure of a hexagonal ice layer in ice $\mathrm{I}_{\mathrm{h}}$.

${ }^{32}$ Gahl C., PhD thesis, Freie Universität Berlin 2004, available online at www.diss.fu-berlin.de/diss/receive/FUDISS_thesis_000000001387.

${ }^{33}$ Bovensiepen U., Gahl C., Wolf M. J. Phys. Chem. B 2003, 107, 8706.

${ }^{34}$ Bovensiepen U., Prog. Surf. Sci. 2005, 78, 87.

${ }^{35}$ Lisowski M., Loukakos P.A., Bovensiepen U., Stähler J., Gahl C., Wolf M. Appl. Phys. A 2004, 78, 165. 
${ }^{36}$ Bovensiepen U., Gahl C., Stähler J., Wolf M. Surf. Sci. 2005, 584, 90.

${ }^{37}$ Kimmel G. A., Petrik N. G., Dohnálek Z., Kay B. D. Phys. Rev. Lett. 2005, 95, 166102.

${ }^{38}$ Haq S. and Hodgson A. J. Phys. Chem. C 2007, 111, 5946.

${ }^{39}$ With increasing ice coverage $>1 \mathrm{BL}$, where crystalline ice islands are formed, we observe a reduction of the 2 PPE intensity $I_{\mathrm{n}=1}$ of the $n=1$ IPS. Thus, the IPS extends from the bare first bilayer in between crystalline ice islands into vacuum. The ratio of areas exhibiting bare first bilayer and crystalline ice islands is determined by normalizing $I_{\mathrm{n}=1}$ to the to the value at $1 \mathrm{BL}$. As the volume is known from the coverage we determine the island height to $4 \mathrm{BL}$ for the mass equivalent coverage of $2 \mathrm{BL}$ shown in Fig. 1. Adding the first bilayer we arrive at a total height of crystalline ice ice structures of $19 \AA$ A.

${ }^{40}$ Stähler J., Mehlhorn M., Bovensiepen U., Meyer M., Kusmierek D.O., Morgenstern K, Wolf M. Phys. Rev. Lett. 2007, 98, 206105.

${ }^{41}$ Meyer M., Stähler J., Wolf M., Bovensiepen U. Phys. Chem. Chem. Phys. 2008, 10, 313002.

${ }^{42}$ Hertwig A., Hippler H., Unterreiner A. N. J. Phys.: Cond. Matter 2000, 12, A 165 (2000); Vilchiz V. H., Kloepfer J. A., Germaine A. C., Lenchenkov V. A., Bradforth S. E. J. Phys. Chem. A 2001, 105, 1711.

${ }^{43}$ Marcus R. A. and Sutin N. Biochim. Biophys. Acta 1985, 811, 265.

${ }^{44}$ Tributsch H., Pohlmann L. Science 1998, 279, 1891.

${ }^{45}$ Kakalios J., Street R. A., Jackson W. B. Phys. Rev. Lett. 1987, 59, 1037.

${ }^{46}$ Phillips J.C. Rep. Prog. Phys. 1996, 59, 1133.

${ }^{47}$ Batista E.R. and Jonsson H. Comp. Mater. Science 2001, 20, 325.

${ }^{48}$ Thierfelder C. , Hermann A., Schwerdtfeger P., and Schmidt W.G. Phys. Rev. B 2006, 74, 045422.

${ }^{49}$ Hermann A., Schwerdtfeger P., Schmidt W. G. J. Phys.: Condens. Matter 2008, 20, 225003.

${ }^{50}$ Santra B., Michaelides A., Scheffler M. J. Chem Phys. 2007, 127, 184104.

${ }^{51}$ In this way we avoid the evaluation of vertical detachment energies that are known (cf. J.M. Herbert and H. Head-Gordon, J. Chem. Phys. A 2005, 109, 5217) to be overestimated by DFT methods compared to sophisticated quantum chemical methods. However, a description of the model system of 96 water molecules outlined below with such ab initio method is presently unavailable. We expect that the trends regarding the trapping of the excess electron and its localization are well captured with the present approach.

${ }^{52}$ Hammer N. I., Shin J.-W., Headrick J. M., Diken E. G., Roscioli J. R., Weddle G. H., Johnson M. A. Science 2004, 306, 675.

${ }^{53}$ Madarász A., Rossky P. J., Turi L. J. Chem. Phys. 2007, 126, 234707.

${ }^{54}$ Kimmel G. A., Petrik N. G., Dohnálek Z., Kay B. D. J. Chem. Phys. 2006, 125, 44713 and references therein.

${ }^{55}$ Stähler J., Meyer M., Kusmierek D.O., Bovensiepen U., Wolf M. J. Am. Chem. Soc. 2008, 130, 8797.

${ }^{56}$ Ryu S., Chang J., Kwon H., Kim S. K., J. Am. Chem. Soc. 2006, 128, 3500.

${ }^{57}$ Bertin M., Meyer M., Stähler J., Gahl. C., Wolf M., Bovensiepen U. Faraday Disc. 2008, 141, DOI: $10.1039 / \mathrm{b} 805198 \mathrm{~d}$. 